\title{
Optimization of ZnS/Ag/ZnS Multilayer Thin Film Systems for Heat Mirror Applications
}

\author{
A. T. Matar \\ Physics Department, Helwan University, Helwan, Cairo, Egypt
}

\begin{abstract}
A multilayer system of dielectric/metal/dielectric $(D / M / D)$ thin films on glass substrate for energy-efficient heat mirror applications were designed theoretically and fabricated experimentally to optimize the performance. The thickness of an Ag film sandwiched between two ZnS layers of specific thickness was considered as the design parameter using IMD-Software. The measured spectral transmittance and reflectance are compared with those computing using relevant software. A good agreement between the theoretical and experimental spectra was obtained. The system performance is optimized by numerical calculations of layers thicknesses then verified experimentally and g/D/M/D device was fabricated. Maximum performance was achieved with Ag thickness in the range of 10 -15 nm and thickness of $15 \mathrm{~nm}$ for each ZnS layer.
\end{abstract}

\section{Introduction:}

The importance and the necessity for energy saving have forced researchers to find a way to reduce the thermal load in hot climate regions in building resulting from solar radiation through the use of thin film coating on the glass windows. On the other hand to enhance its transmission for heating purposes as it is required in cold climates regions. Dielectric/metal/dielectric (DMD) multilayers film has been extensively investigated as an important low emittance configuration for infrared radiation (heat mirror) [1].

For a glass window to be energy-efficient in warm climates, it should have spectrally selective coatings such that it transmits the energy in the visible (VIS) and reflects all the energy in the infrared (NIR); heat mirror performance. Three-layer systems of dielectric/metal/dielectric (D/M/D) coated on glass substrates have been used as a spectrally selective coating for various purposes [2-7]. The highly IR reflective metal film, that transmits very little energy in the visible, was sandwiched between the two dielectric layers that act as antireflective coatings to enhance the energy transmitted in the visible region. By varying the material and thickness of one or more of the three layers, the optical behaviour of the multilayer $\mathrm{D} / \mathrm{M} / \mathrm{D}$ device can be tailored to suit different applications. 
The objective of the present work is the use of numerical calculation for optimization the layers thicknesses of high performance glass/D/M/D device for heat mirror applications. The thickness of a silver film sandwiched between two zinc sulfide layers of specific thickness will be considered using IMD-Software [8] for modeling the optical properties of multilayer films. The calculated results have to be experimentally verified.

\section{Theory:}

Consider a plane electromagnetic wave incident with an angle $\theta_{\mathrm{i}}$ on an interface of multilayer stacking as shown in Fig. (1) with $N$ layers and $N+1$ interfaces, where the $i^{\text {th }}$ layer has thickness $d_{i}$ and complex optical index of refraction $\mathbf{n}_{i}(=n+i k$, where $n$ is the refractive index and $k$ is the extinction coefficient). The regions above the multilayer stack (the ambient) and below it (the substrate) have the refractive indices $\mathbf{n}_{\mathrm{a}}$ and $\mathbf{n}_{\mathrm{s}}$, respectively. The case of a free-standing film refers to the condition $\mathbf{n}_{\mathrm{s}}=\mathbf{n}_{\mathrm{a}}$. Under these circumstances, the net reflection $\left(r_{i}\right)$ and transmission $\left(t_{i}\right)$ coefficients of the $i^{\text {th }}$ layer are given as [9],

$$
\begin{aligned}
& r_{i}=\frac{r_{i j}+r_{j} e^{2 i \beta_{i}}}{1+r_{i j} r_{j} e^{2 i \beta_{i}}} \\
& t_{i}=\frac{t_{i j} t_{j} e^{2 i \beta_{i}}}{1+r_{i j} r_{j} e^{2 i \beta_{i}}}
\end{aligned}
$$

where $\beta_{\mathrm{i}}=2 \pi d_{i} \mathbf{n}_{i} \cos \theta_{i} / \lambda$ and $r_{i j}$ and $t_{i j}$ are given by the well-known Fresnel equations while the reflection and transmission coefficients $r_{i j}$ and $t_{i j}$, respectively, are computed according to Stearns [10]. Although the roughness in the layers of the multilayer stack can be considered in the formalization developed by Stearns [10] to compute the net reflection $(r)$ and transmission $(t)$ coefficients, it is ignored here. Physics and algorithms used for modeling and parameter calculations (theoretical bases as well as the final equations) are given in details by Windt [8]. The reflectance, $R$ and transmittance, $T$, which measure the energy reflected from or transmitted through the film, respectively, are then given by:

$$
R=|r|^{2}
$$

and

$$
\mathrm{T}=\left[\frac{\mathrm{n}_{\mathrm{s}} \cos \theta_{\mathrm{s}}}{\mathrm{n}_{\mathrm{a}} \cos \theta_{\mathrm{a}}}\right]|\mathrm{t}|^{2}
$$




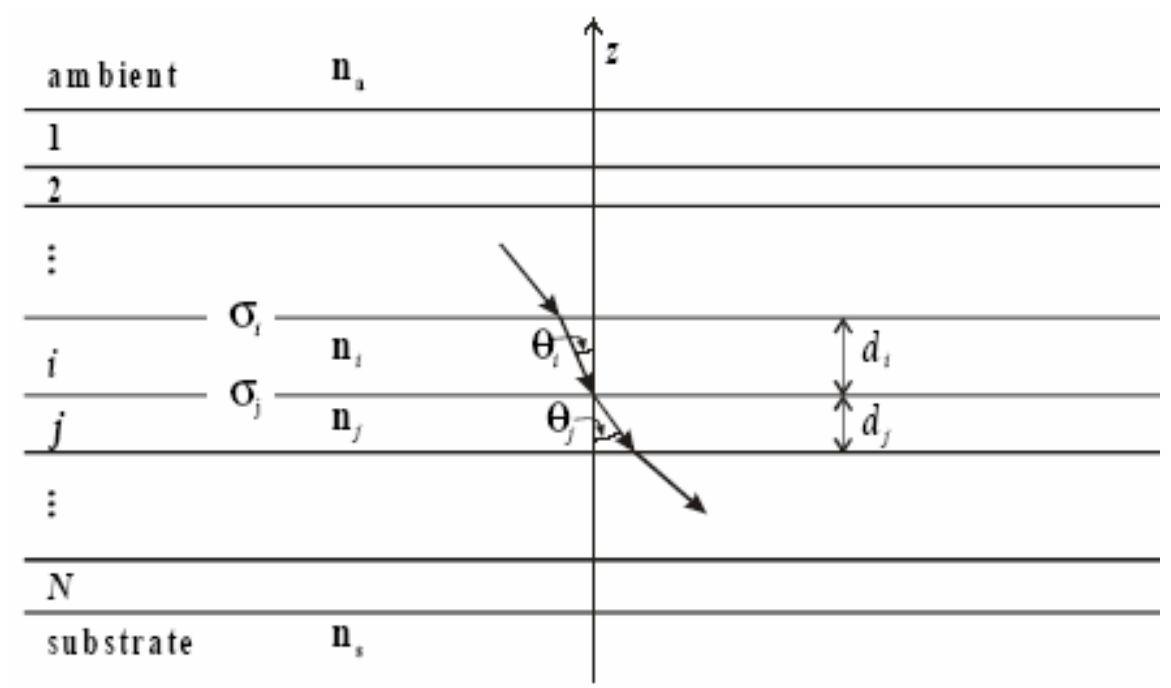

Fig. (1): Stacked multilayer system.

\section{Experimental and Computational Work:}

\subsection{Sample Preparation:}

The multilayer ZnS/Ag/ZnS films were prepared using electron-gun under a vacuum of $\left(10^{-5}\right.$ torr) obtained in a Laybold-Heraeus vacuum system Molybdenum boats were used where the $\mathrm{ZnS}$ and Ag were sublimated at a low deposition rate of $0.1 \mathrm{~nm} / \mathrm{s}$. The thickness and deposition rate of the prepared films were estimated roughly by the quartz crystal thickness monitor model INFICON-XTM. For accurate thickness values the monitor was calibrated interferometrically by multiple-beam Fizeau fringes of equal chromatic order at reflection. Thickness of $15 \mathrm{~nm}$ for $\mathrm{ZnS}$ was chosen to achieve thin and continuous film while that of Ag was the modeled optimum value.

\subsection{Optical Measurements:}

The optical transmittance $\mathrm{T}$ (normal incident) and reflectance R (incident angle of $5^{\circ}$ ) of unpolarized radiation were recorded over the wavelength range 300-2500 nm, using a double beam spectrophotometer (Shimadzu UV-3101 PC: UV-VIS-NIR). 


\subsection{Computation:}

Computations of the spectral $\mathrm{T}$ and $\mathrm{R}$ were carried out on a multilayer stack of $\mathrm{ZnS} / \mathrm{Ag} / \mathrm{ZnS}$ coated on an amorphous glass $\left(\mathrm{SiO}_{2}\right)$ substrate for wavelengths ranging from $300 \mathrm{~nm}$ to $2500 \mathrm{~nm}$. While ZnS film thickness was keep constant at $15 \mathrm{~nm}$ through out the computation, the Ag film thickness was varied from 5 to $35 \mathrm{~nm}$ in steps of $5 \mathrm{~nm}$. Although variation in the experimental values of the optical constants with the preparation parameters is expected, the refractive indices in the considered wavelengths that are taken from the data stored in the IMD program [8] meet the needs in this theoretical computation. Each optical constants file contains the optical data $(\lambda, n$ and $k$ ) associated with a single material.

IMD is used for calculating specular and non-specular (diffuse) optical functions of an arbitrary multilayer structure, i.e., a structure consisting of any number of layers of any thickness and of any material. Computations of the optical functions of a multilayer film in IMD are based on application of the Fresnel equations, modified to account for interface imperfections, which describe the reflection and transmission of an electromagnetic plane wave incident at an interface between two optically dissimilar materials. The overall transmission and reflection coefficients were calculated according to the following equation,

$$
\chi=\frac{\int \phi(\lambda) \chi(\lambda) \mathrm{d} \lambda}{\int \phi(\lambda) \mathrm{d} \lambda}
$$

where: $\phi(\lambda)$ is the photopic sensitivity from table in VIS and is the spectral intensity distribution of a blackbody at $300 \mathrm{~K}$ in NIR.

$\chi(\lambda)$ is the spectral distribution of $\mathrm{T}$ or $\mathrm{R}$.

$\chi$ is the overall value of $\mathrm{T}$ or $\mathrm{R}$ in the wavelength $(\lambda)$ range of VIS $=$ $380-780 \mathrm{~nm}$ and NIR $=780-2000 \mathrm{~nm}$.

Since the performance of a heat mirror depends on both the transmission and reflection coefficients, thus, a performance coefficient has been defined [11] as:

$$
P C=\frac{T_{V I S} R_{N I R}}{T_{N I R} R_{V I S}+0.01}
$$

The optimum condition is specified by plotting PC versus Ag layer thickness. 


\section{Results and Discussion:}

\subsection{Performance Optimization:}

The spectral transmittance $T(\lambda)$ and reflectance $R(\lambda)$ of $\mathrm{ZnS} / \mathrm{Ag} / \mathrm{SnS}$ multilayer device was theoretically calculated for fixed ZnS layer thickness of $15 \mathrm{~nm}$ and different values of Ag layer thickness. The computer simulated transmittance and reflectance are presented in Fig. (2) within the wavelength range from 300 to $2500 \mathrm{~nm}$ for different Ag thickness from 5 to $35 \mathrm{~nm}$. A considerable variation was observed in spectral properties (both $\mathrm{T}$ and $\mathrm{R}$ ). Generally, as the thickness of Ag layer has been increased, $T(\lambda)$ is reduced while $R(\lambda)$ is enhanced. Also, the wavelength, at which $T(\lambda)$ is maximum is shifted towards the shorter wavelength and centered on the VIS region at Ag thickness of about $10 \mathrm{~nm}$. However, $(\mathrm{R}+\mathrm{T})$ is equal unity in all cases where the absorption is neglected.

It is worth mentioning that, the highest performance of heat mirrors depends on the values of both $T(\lambda)$ in the VIS range and $R(\lambda)$ in NIR one. Therefore, the performance was estimated according to Eqn. (13) that includes the integrated values $T_{V I S}$ and $R_{N I R}$ in the required wavelength ranges. The dependence of the calculated performance coefficient (PC) on the thickness of Ag layer for theoretically modeled device is depicted in Fig.(3). It is clear that there is a great variation in the performance depending on the Ag film thickness with a maximum at thicknesses between 10 and $15 \mathrm{~nm}$. The performance shows a gradual decrease on the side of larger Ag thickness than $15 \mathrm{~nm}$ while it considerably deteriorates as the Ag thickness decreases than $10 \mathrm{~nm}$.

\subsection{Experimental Verification:}

The above theoretical modelling has to be verified experimentally. From the behaviour of performance-dependence on Ag film thickness, a case on the larger thickness side in the range of maximum, but not fare, that is of $15 \mathrm{~nm}$ was considered for experimental verification. Fig. (4) represents the theoretically simulated as well as the experimentally obtained data of $T(\lambda)$ and $R(\lambda)$ for the same thicknesses of layers. Although $(T+R)<1.0$ in the experimental case due to absorption, there is a good agreement between the two cases.

In order to test the goal of the present approach, the performance coefficient was calculated from the experimental data and presented in Fig. (3) for comparison. A satisfactory coincidence of both the theoretical prediction and the experimental results is achieved. This confirms the capability of the computer simulation and the used program for modelling $\mathrm{D} / \mathrm{M} / \mathrm{D}$ devices for application in solar selective coatings. 

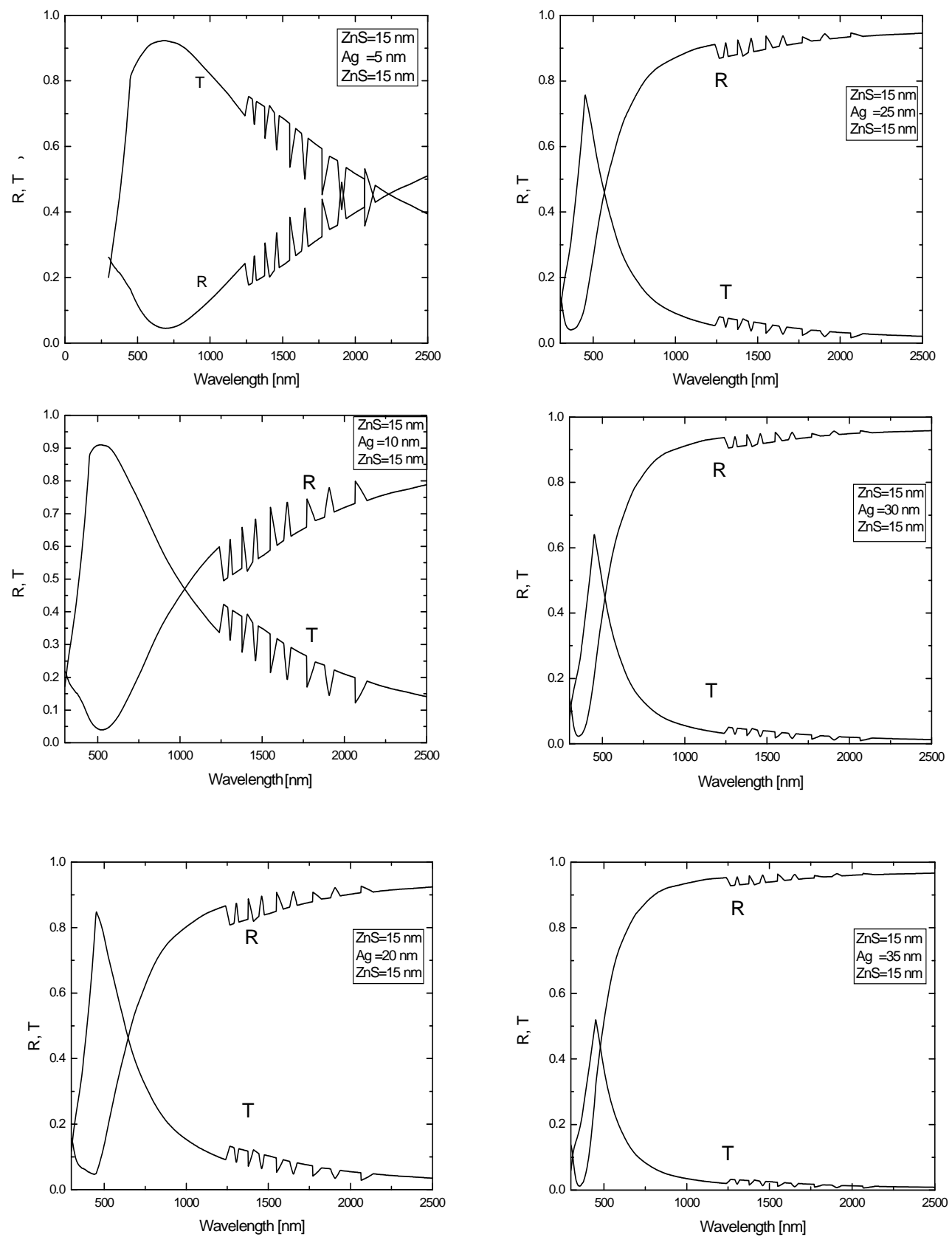

Fig. (2): Theoretically calculated spectral transmittance and reflectance for $\mathrm{ZnS}$ thickness of $15 \mathrm{~nm}$ with different Ag film thickness. 


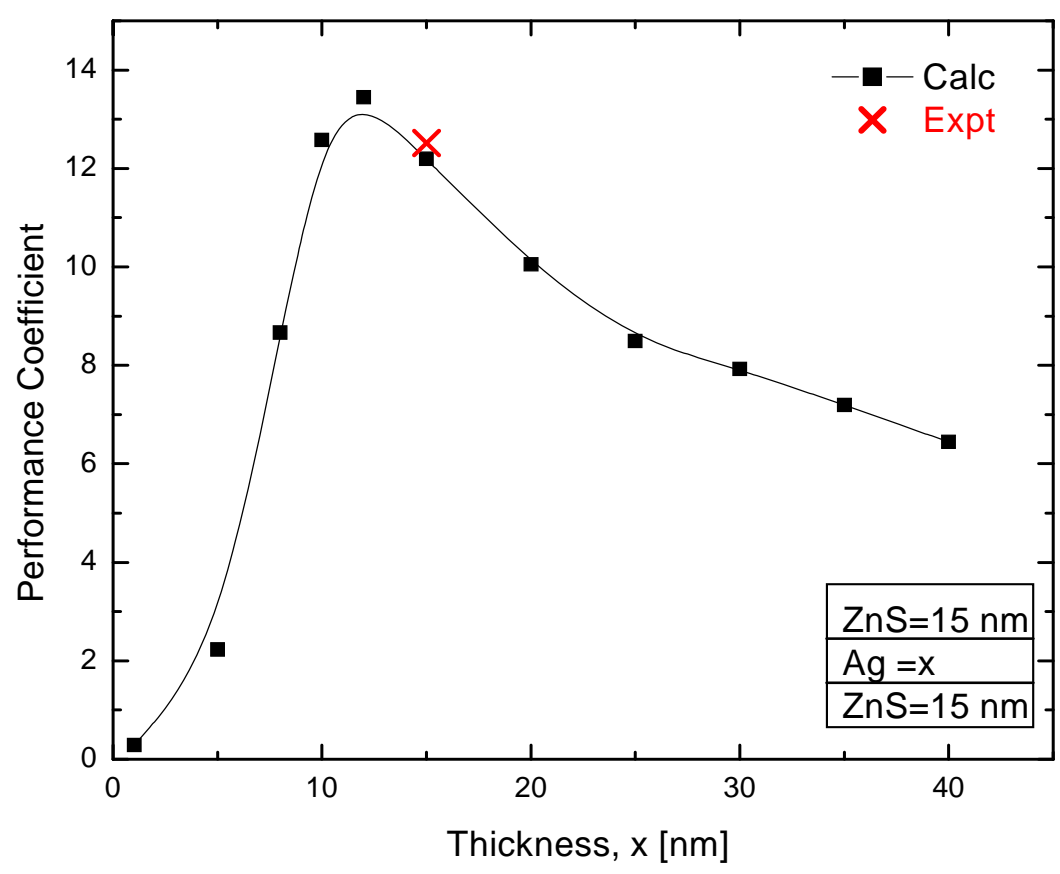

Fig. (3): The performance coefficient PC as a function of Ag film thickness $x$

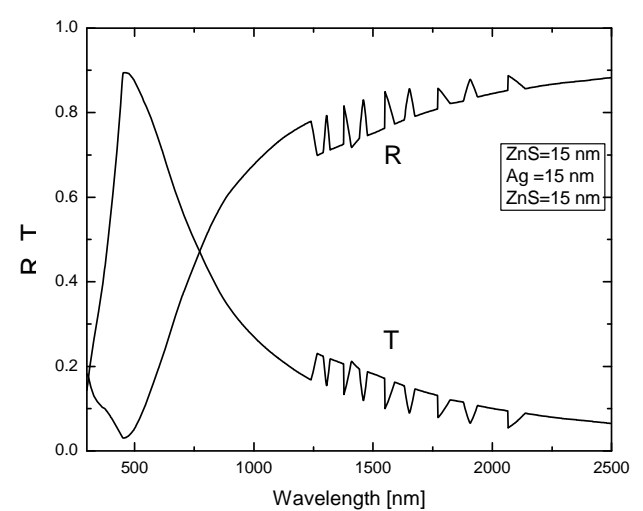

(a)

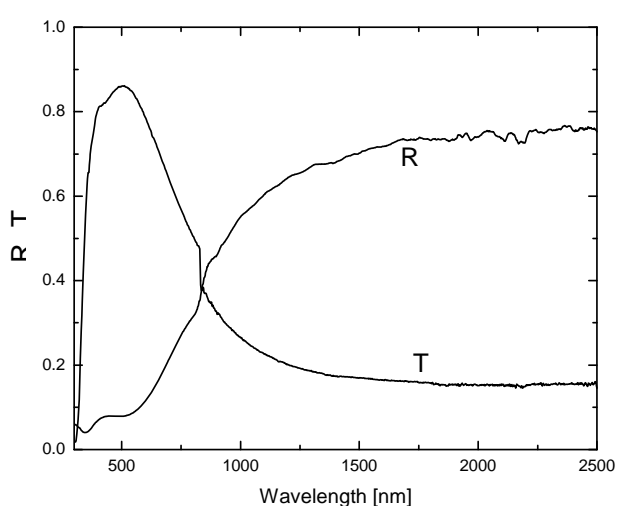

(b)

Fig. (4): Spectral transmittance and reflectance for $\mathrm{ZnS}$ film of $15 \mathrm{~nm}$ thickness and Ag film of $15 \mathrm{~nm}$ thickness: a) theoretically and b) experimentally obtained data. 


\section{Conclusions:}

For materials and time saving, theoretical modelling is a recommended as useful approach to fabricate multilayer devices for selective solar coating applications. Experimental verification of the data obtained by a computational program is needed. The optical constants, which are stored in the (IMD) program, can be used safely in the theoretical modeling. High performance of $\mathrm{ZnS} / \mathrm{Ag} / \mathrm{ZnS}$ thin films heat mirror can be achieved with Ag layer of $12 \mathrm{~nm}$ thick sandwiches between two ZnS layers of $15 \mathrm{~nm}$ each.

\section{References:}

1. R.B. Goldner. J. Vac. Sci. Technol. A 13, 1088 (1995).

2. J.A. Pracchia and J.M. Simon. Appl. Opt. 20, 251 (1981).

3. C.G. Granqvist. Appl. Opt. 20, 2606 (1981).

4. H. Köstlin and G. Frank. Thin Solid Films 89, 287 (1982).

5. C.C. Lee, S.H. Chen and C.C. Jaing. Appl. Opt. 35, 5698 (1996).

6. X. Zhang, S. Yu and M. Ma. Sol. Energy Mater. Sol. Cells 44, 279 (1996).

7. G. Leftheriotis, P. Yianoulis and D. Patrikios. Thin Solid Films 306, 92 (1997).

8. David L. Windt, Computers in Physics, 12 (4), 360 (1998).

9. O. S. Heavens, "Optical Properties of Thin Solid Films", Dover Publications, New York, (1991).

10. D. G. Stearns, J. Appl. Phys. 65, 491 (1989).

11. H. M. Hashem, Ph. D. Thesis, Physics Department, Helwan University, Egypt (2008). 